\title{
VIRTUAL COHOMOLOGICAL DIMENSION OF MAPPING CLASS GROUPS OF 3-MANIFOLDS
}

\author{
DARRYL MCCULLOUGH
}

The mapping class group of a topological space is the group of self-homeomorphisms modulo the equivalence relation of isotopy. For 2-manifolds (of finite type), it is a discrete group which is known (see [M, H1, H2, H3, H4]) to share many of the properties of arithmetic subgroups of linear algebraic groups, although it is not arithmetic. In this note we describe the results of [M1], which show that the mapping class groups of many 3-manifolds share some of these properties.

More precisely, a group $G$ is said to be of type $F L$ if the trivial $G$-module $\mathbf{Z}$ admits a resolution of finite length by finitely generated free $G$-modules (see [S]), and is said to be a duality group when there is a $G$-module $C$ such that cap product with an element $e \in H_{n}(G ; C)$ induces isomorphisms $H^{k}(G ; A) \cong H_{n-k}(G ; C \otimes A)$ for all $k$ and $A$ (see [B-E]). The classes of groups of type FL and duality groups are closed under extension. The main result of [M1] is

THEOREM 1. Let $M$ be a compact orientable irreducible sufficiently large 3-manifold. Then the mapping class group $\mathcal{H}(M)$ is finitely presented and contains a subgroup of finite index which is of type FL. If the boundary of $M$ is incompressible, then the subgroup is a duality group.

The finite presentation of $\mathcal{H}(M)$ in the boundary-incompressible case follows from work of Johannson [J] and Hemion [H5] (see [W]). In the case of compressible boundary, it was proved by R. Kramer for handlebodies and by P. Grasse [G] in general.

A finitely presented group of type FL is also called a geometrically finite group because it is the fundamental group of a finite aspherical complex. Its cohomology, with any coefficient module, vanishes above a certain dimension, called the cohomological dimension of the group.

When a group $G$ contains a subgroup of finite index which has finite cohomological dimension, $G$ is said to have finite virtual cohomological dimension. This dimension is well defined (see $[\mathbf{S}]$ ) and is denoted by $\operatorname{dim}(G)$.

1. The proof of Theorem 1. The characteristic submanifold theory due to Johannson $[\mathbf{J}]$ and Jaco and Shalen $[\mathbf{J}-\mathbf{S}]$ shows that Haken 3-manifolds

Received by the editors July 27, 1987.

1980 Mathematics Subject Classification (1985 Revision). Primary 57M99; Secondary 57S99, 55S37, 20F34.

Key words and phrases. 3-manifold, mapping class group, homeomorphism, diffeomorphism, cohomological dimension, duality group, FL, VFL.

Supported in part by NSF Grants DMS-8420067 and DMS-8701666. 
(that is, manifolds as in Theorem 1 having incompressible boundary) consist of invariant (up to isotopy) pieces which are either Seifert fibered, $I$-fibered, or "simple"; the latter have finite mapping class groups $[\mathbf{J}]$ and are negligible when considering virtual properties of $\sharp(M)$. For a Seifert fibered 3-manifold $\Sigma$, apart from a few exceptional cases which can be handled explicitly, the orientation-preserving mapping class group $\mathfrak{H}_{+}(\Sigma)$ is isomorphic to the group of orientation-preserving fiber-preserving mapping classes $\mathcal{H}_{+}^{f}(\Sigma)$. From $[\mathbf{J}$, Propositions 25.2 and 25.3], excepting some more cases, there is an exact sequence

$$
1 \rightarrow H_{1}(F, \partial F) \rightarrow \mathcal{H}_{+}^{f}(\Sigma) \rightarrow \mathcal{H}^{*}(F) \rightarrow 1
$$

in which $F$ is the orbit surface of $\Sigma$ and $H^{*}(F)$ is a subgroup of finite index in $\mathcal{H}\left(F^{\prime}\right)$, where $F^{\prime}$ is the result of removing from $F$ the points which correspond to exceptional orbits of $\Sigma$. The kernel $H_{1}(F, \partial F)$ is isomorphic to the group of "vertical" mapping classes that map each fiber to itself. Work of Harer [H1], which we extend to nonorientable 2-manifolds, shows that $\mathcal{H}^{*}(F)$ is a virtual duality group, and the intersection of the vertical mapping classes with a certain subgroup of finite index in $H(\Sigma)$ is a finitely generated free abelian group. This proves Theorem 1 for the Seifert fibered case. For $I$-bundles, the mapping class group is essentially the same as the mapping class group of the orbit surface, and Theorem 1 follows from the 2-dimensional version.

For the general case of incompressible boundary, there is a subgroup of finite index in $\mathcal{H}(M)$ which maps onto the product of (certain subgroups of) the mapping class groups of the components of its characteristic submanifold, with kernel the finitely generated abelian subgroup generated by Dehn twists about the components of the frontier of the characteristic submanifold. Somewhat surprisingly, this kernel can contain torsion, and some effort is required to find a finite index subgroup of $\mathcal{H}(M)$ that avoids this torsion. These ideas combine to yield Theorem 1 in the Haken case.

In the case of compressible boundary, there is a third kind of characteristic piece called a product-with-handles, studied in $[\mathbf{B}]$ and $[\mathbf{M}-\mathbf{M}]$. For a product-with-handles $V$, there is a simplicial complex $L$ in which the vertices are the isotopy classes of essential compressing discs in $V$, and a collection of vertices spans a simplex if and only if the isotopy classes can be represented by a collection of pairwise disjoint discs in $V$. We prove that $L$ is a finitedimensional contractible complex admitting a simplicial action of $\mathcal{H}(V)$ with finite quotient. Since the result of cutting a product-with-handles along a set of compressing discs is a collection of products-with-handles of lower complexity, it is possible to analyze the stabilizers of simplices in $L$ inductively, obtaining enough information to establish that $\mathcal{H}(V)$ is finitely presented and virtually of type FL. The general case follows, using the techniques for working with 3-manifolds with compressible boundary developed in [M-M].

For the cases when the boundary of $M$ is compressible $I$ do not know in general whether $\nvdash(M)$ is a virtual duality group. For the genus 2 handlebody, we have

THEOREM 2. Let $V$ denote the orientable handlebody of genus 2 . Then $H(V)$ is a virtual duality group of dimension 3. 
The proof uses a theorem of R. Kramer [K] which describes $\not_{+}(V)$ as a free product with amalgamation $A *_{C} B$. Investigation shows that $A, B$, and $C$ are virtual duality groups of dimension 3 , and Theorem 2 is proved by showing that the subgroup of $H(V)$ which acts trivially on $H_{1}(V ; \mathbf{Z} / 3)$ is a graph product all of whose vertex and edge groups are duality groups of dimension 3.

Kramer's theorem suggests that the genus 2 case is very special, and may be atypical.

2. Calculation of virtual cohomological dimension. The work in [H1] and its extension to the nonorientable case give the precise virtual cohomological dimension of the mapping class groups of 2-manifolds of finite type. In the 3-dimensional case, we consider first the manifolds which can be components of the characteristic submanifold. For an irreducible $I$-bundle $\Sigma$ over $F$, we have $\operatorname{dim}(H(\Sigma))=\operatorname{dim}(H(F))$.

PROPOSITION 3. Let $\Sigma$ be a Haken 3-manifold which is Seifert fibered over $F$. Define $F^{\prime}$ to be the complement of the exceptional points in $F$.

(a) If $\Sigma$ is an $S^{1}$-bundle over the torus with nonzero Euler class, then $\operatorname{dim}(\not{H}(\Sigma))=1$.

(b) If $\Sigma$ is an $S^{1}$-bundle over the Klein bottle with nonzero Euler class, then $\operatorname{dim}(\mathcal{H}(\Sigma))=0$.

(c) In all other cases, $\operatorname{dim}(\mathcal{H}(\Sigma))=\operatorname{rank}\left(H_{1}(F, \partial F)\right)+\operatorname{dim}\left(\mathcal{H}\left(F^{\prime}\right)\right)$.

Apart from a few exceptional cases which must be handled individually, this follows from the exact sequence $(*)$ above.

When $M$ is simple, $\mathcal{H}(M)$ is finite by [J, Theorem 27.1], so $\operatorname{dim}(\mathcal{H}(M))=0$.

For the general Haken case, some definitions are required. If $\Sigma_{i}$ is a component of the characteristic submanifold, denote by $\widehat{F_{i}^{\prime}}$ the result of attaching a 2-disc to each component of $\partial F_{i}^{\prime}$ that is the image of a component of the frontier of $\Sigma_{i}$ that is a torus. Let $\mathcal{H}^{\prime}\left(F_{i}^{\prime}\right)$ denote the group of mapping classes of homeomorphisms that preserve the image of the frontier of $\Sigma_{i}$.

THEOREM 4. Let $M$ be a Haken 3-manifold which is not I-fibered, Seifert fibered, or simple. Let $\Sigma_{1}, \Sigma_{2}, \ldots, \Sigma_{n}$ be the components of the characteristic submanifold $\Sigma$ of $M$, and let $S_{1}, S_{2}, \ldots, S_{m}$ be the components of the closure of $M-\Sigma$. Let $t$ be the number of components of the frontier of $\Sigma$ that are tori and let $a$ be the number that are annuli. Then

$$
\begin{aligned}
\operatorname{dim}(\mathcal{H}(M))= & 2 t+a+k \\
& -\sum_{i=1}^{n} \operatorname{rank}\left(\operatorname{center}\left(\pi_{1}\left(\Sigma_{i}\right)\right)\right)-\sum_{j=1}^{m} \operatorname{rank}\left(\operatorname{center}\left(\pi_{1}\left(S_{j}\right)\right)\right) \\
& +\sum_{i=1}^{n} \operatorname{dim}\left(\mathcal{H}^{\prime}\left(F_{i}^{\prime}\right)\right)+\sum_{\left\{i \mid \Sigma_{i} \text { is Seifert-fibered }\right\}} \operatorname{rank}\left(H_{1}\left(\widehat{F_{i}^{\prime}}, \partial \widehat{F_{i}^{\prime}}\right)\right),
\end{aligned}
$$

where $k$ is the number of components of $\Sigma$ that are homeomorphic to $S^{1} \times S^{1} \times I$ and contain a component of the frontier of $\Sigma$ which is an annulus.

For the case of handlebodies, an inductive argument yields the following estimate. 
THEOREM 5. Let $V_{g}$ be a handlebody of genus $g \geq 2$. Then $\operatorname{dim}\left(\mathcal{H}\left(V_{2}\right)\right)=$ 3 , and if $g \geq 3$, then $3 g-2 \leq \operatorname{dim}\left(\mathcal{H}\left(V_{g}\right)\right) \leq 4 g-5$.

A similar estimate can be made for products-with-handles.

\section{REFERENCES}

[B-E] R. Bieri and B. Eckmann, Groups with homological duality generalizing Poincaré duality, Invent. Math. 20 (1973), 103-124.

[B] F. Bonahon, Cobordism of automorphisms of surfaces, Ann. École Norm. Sup. (4) 16 (1983), 237-270.

[G] P. Grasse, Results on finite presentation of mapping class groups of certain 3-manifolds, dissertation, Univ. of Oklahoma, 1986.

[H1] J. Harer, The virtual cohomological dimension of the mapping class group of an orientable surface, Invent. Math. 84 (1986), 157-176.

[H2] , The cohomology of the moduli space of curves, preprint.

[H3] W. Harvey, Geometric structure of surface mapping class groups, Homological Group Theory (C. T. C. Wall, ed.), London Math. Soc. Lecture Note Series No. 36, Cambridge Univ. Press, Cambridge and New York, 1979, pp. 255-269.

[H4] _ Boundary structure of the modular group, Riemann Surfaces and Related Topics: Proceedings of the 1978 Stony Brook Conference, Ann. Math. Studies, No. 97, Princeton Univ. Press, Princeton, N.J., 1981, pp. 245-251.

[H5] G. Hemion, On the classification of homeomorphisms of 2-manifolds and the classification of 3-manifolds, Acta Math. 142 (1979), 123-155.

[J-S] W. Jaco and P. Shalen, Seifert fibered spaces in 3-manifolds, Mem. Amer. Math. Soc. no. 220, 1979, pp. 1-192.

[J] K. Johannson, Homotopy equivalences of 3-manifolds with boundaries, Lecture Notes in Math., vol. 761, Springer-Verlag, Berlin and New York, 1979.

[K] R. Kramer, The twist group of an orientable cube-with-two-handles is not finitely generated, unpublished.

[M] J. McCool, Some finitely presented subgroups of the automorphism group of a free group, J. Algebra 35 (1975), 205-213.

[M1] D. McCullough, Virtually geometrically finite mapping class groups of 3-manifolds, preprint.

[M-M] D. McCullough and A. Miller, Homeomorphisms of 3-manifolds with compressible boundary, Mem. Amer. Math. Soc. no. 344, 1986, pp. 1-100.

[S] J.-P. Serre, Cohomologie des groupes discrets, Prospects in Mathematics, Ann. of Math. Studies no. 70, Princeton Univ. Press, Princeton, N.J., 1971, pp. 77-169.

[W] F. Waldhausen, Recent results on sufficiently large 3-manifolds, Proc. Sympos. Pure Math., vol. 32, Amer. Math. Soc., Providence, R.I., 1978, pp. 21-38.

Department of Mathematics, University of OKLahoma, Norman, OKLAHOMA 73019 